outcome was defined as 1-3 months modified Rankin Scale (mRS). All patients were divided into three group based on their follow up mRS. Patients with mRS 0-2, patients with mRS 3-5 and patients with mRS 6 .

Results A total of 66 patients with stenosis or occlusion of posterior circulation presenting with acute ischemic stroke were identified. Out of 66, complete imaging review of 52 patients was completed. Out of 52, no BAO occlusion or high grade stenosis was identified in 11 patients on vascular imaging. In 8 patients, there was no follow up mRS score or baseline vascular imaging available. Thirty two patients were included in the final analysis, out of which, 10 patients were mRS 0-2 group, 6 patients were in mRS 3-5 group and 16 patient were in mRS 6 group (see Table 1). Mean age in both groups was $64.7,51.6$ and 69.2 years respectively with male predominance. Hypertension was the most common baseline comorbidity in all groups. In mRS 6 group, 37.5\% had occlusion in vertebrobasilar junction. Thrombectomy was performed in $40 \%, 33.3 \%$ and $37.6 \%$ patients repectively. The last known well (LKW) to groin puncture time was increased in patients with mRS 6 .

Conclusion This retrospective analysis of BAO database depicts that older age, presence of HTN, history of prior stroke, location of clot at vertebrobasilar junction, and absence of bilateral posterior communicating arteries is associated with higher mortality. Moreover, delayed time to groin puncture was also observed in patients with higher mortality. Large scale studies are needed to validate these findings.

Disclosures A. Zafar: None. S. Suriya: None. M. Farooqui: None. A. Ikram: None. S. Ortega-Guiterrez: None. M. Torbey: None.

\section{E-103 SHORT-TERM IN-HOSPITAL OUTCOMES OF THROMBOLYSIS FOR ACUTE ISCHEMIC STROKE PATIENTS WITH NON-PRIMARY BRAIN TUMORS AND CONGESTIVE HEART FAILURE}

K Tong*, T Colburn, P Brauer. Anatomy, Kansas City University of Medicine and Biosciences, Kansas City, MO

\subsection{6/neurintsurg-2019-SNIS.178}

Background Intravenous thrombolysis remains an underutilized treatment for acute ischemic stroke (AIS) due to several relative and absolute contraindications. Previous studies have found similar outcomes after thrombolysis between AIS patients with benign brain tumors and AIS patients without. This study aims to investigate short-term outcomes of thrombolytic treatment for the greater majority of AIS patients who have no history of primary brain tumors, particularly those with congestive heart failure (CHF).

Methods This retrospective cohort study utilized data from the 2012-2015Q3 Nationwide Inpatient Sample (NIS). ICD-9 codes identified adult patients (ages $18+$ ) who suffered acute ischemic stroke and received intravenous thrombolysis, and then further isolated patients diagnosed with CHF. Data for patients who were missing important clinical identifiers (age, gender, race, mortality), did not receive IV thrombolysis, and had primary brain tumors (benign or malignant) were excluded. Data analyses assessed hospital mortality rate, length of stay (LOS), inpatient charges, and average age of admission.
Results Of the 24,692 encounters with AIS patients treated with thrombolysis and had no history of primary brain tumors, 4130 were diagnosed with CHF.

- Mean mortality rate was significantly increased $(11.3 \% \mathrm{CHF}$ vs. $11.8 \%$ no $\mathrm{CHF}, \mathrm{p}<0.0001$ ).

- Mean LOS was significantly longer (7.63 days CHF vs. 6.08 days no CHF, $\mathrm{p}<0.0001)$.

- Average total charges were significantly increased $(\$ 96,978.03$ CHF vs. \$83,905.14 no CHF, $\mathrm{p}<0.0001)$.

- Average age at admission was significantly older (70.68 years CHF vs. 66.32 years no CHF, $\mathrm{p}<0.0001)$.

Conclusion This study aims to inform physicians to better manage AIS patients receiving IV thrombolysis with $\mathrm{CHF}$ and no history of primary brain tumors. These patients experience higher mortality rate, longer LOS, increased total hospital charges, and older age at admission than those without CHF. These findings suggest that placing clinical focus on the coexisting condition of CHF before administering IV thrombolysis for AIS may be critical for improving short-term in-hospital outcomes. Future research should aim to investigate different thrombolytic agents to determine the most optimal choice for patients with congestive heart failure.

Disclosures K. Tong: None. T. Colburn: None. P. Brauer: None.

\section{E-104 THE USE OF MECHANICAL THROMBECTOMY IN POSTERIOR CIRCULATION ACUTE ISCHEMIC STROKES}

A Sweid*, K Hafazalla, S Tjoumakaris, V Xu, K Shivashankar, T Alexander, M Gooch, N Herial, N Chalouhi, R Rosenwasser, P Jabbour. Neurosurgery, Thomas Jefferson University, Philadelphia, PA

\subsection{6/neurintsurg-2019-SNIS.179}

Objective 20\% of all acute ischemic strokes (AIS) occur in the posterior circulation (PC), which carry a high mortality rate close to 95\%. Early recanalization improves outcomes as shown by several reports. Often trials excluded patients with posterior circulation strokes. We sought to investigate the prevalence, safety, and the clinical outcomes of PC-MT at our institution.

Methods A retrospective review of patients presenting with PC AIS who underwent mechanical thrombectomy at a tertiary referral center between 2010 and 2018.

Results Out of 453 patients who underwent mechanical thrombectomy for AIS, 45 patients had a PC stroke (av age 58 years, 56\% male). The mean NIHSS upon admission was 17 (95\%CI:14-20). The mean time from symptoms onset to groin puncture was 9 hours (95\%CI:6-11), the mean procedure time was 53 minutes (95\%CI:43-64) and mean time to revascularization, from symptoms onset was 866 minutes (95\%CI:602-1129). Only 33\% received tPA. Vessels involved included the basilar artery $(87 \%, \mathrm{n}=39)$, the posterior cerebral artery $(4 \%, n=2)$, and the vertebral artery $(9 \%, n=4)$. The procedures were divided into stentreiver alone $(22 \%, n=10)$, aspiration alone $(13 \%, \mathrm{n}=6)$, and stentreiver with aspiration $(51 \%, n=23)$, and failed procedure in $13 \%(n=6)$ of individuals. Most procedures required one pass to recanalize the vessel $(44 \%, n=20)$. TICI $\geq 2 \mathrm{~b}$ was achieved in $69 \%$ of the subjects. Mean length of stay was 11 days (95\%CI:8-14). Mortality rate was $38 \%$ and independent functional outcome of 